and four weeks before admission paresis of the lower limbs began, with numbness and tingling. These symptoms increased gradually, later affecting the upper limbs also, though to a less extent. An attack of gastric disturbance with jaundice shortly preceded admission.

The previous health and family history were satisfactory. There was no history of alcoholism or of any other possible cause for her symptoms except the influenza.

Under treatment by massage there was rapid diminution of the œdema and gradual improvement in regard to the pain, and gradual return of muscle power. The massage was kept up for ten weeks, by which time the patient was able to walk about the ward, and she went home almost

This patient's illness corresponded with the usual features of alcoholic peripheral neuritis, and there was at first a suspicion that it might be of that nature. However, her own assurances on this point were amply corroborated by the members of her own family, by her employer, and by her physician. Moreover, although she had had a distinct attack of influenza, she did not lay up for any time, and against medical advice persevered at her work, so that there was all the more likelihood of such a complication as neuritis taking place. Cases might be multiplied either from the wards of the infirmary or from private case books of other forms of nervous disease of a chronic nature-the second group in Dr. Bury's paper-which are now recognised to be not very infrequent sequelæ of influenza, but I content myself with narrating the above as a recent and very typical case.

I am indebted to Sir William Gairdner for the suggestion to record these cases, and for his kind help in the preparation of this paper. The original reports of the cases were all the work of Dr. A. L. Watson, recently Resident Physician for Sir William in the Western Infirmary, and it is due to him to acknowledge here the careful and thorough way in which they were recorded.

\section{THE DETECTION OF ARSENIC IN BEER AND BREWING MATERIAL.}

BY SHERIDAN DELÉPINE, M.B., Procter Professor of Pathology, Owens College, Manchester.

Tнe preliminary note contributed by Dr. Tattersall and myself $^{1}$ had for its object to prevent the further use in brewing of some sugars which we had found to be the sole cause of the dangerous contamination of beers brewed in Salford. Dr. Tattersall's preliminary report to his Committee dealt only with this practical aspect of the question, a more detailed account of the work done being reserved for a more complete report. The announcements and suggestions which continue to be made have convinced us of the desirability of publishing a short account of the methods of analysis which has been selected, and of the considerations which have led me to adopt certain standards for the purpose of interpreting the results obtained.

The Wide Distribution of Arsenic in Nature.

In the first place, I had to keep in mind the extraordinary ubiquity of arsenic. It is a fact of common knowledge among chemists ${ }^{2}$ and biologists ${ }^{3}$ that arsenic is one of the most widely-distributed substances. It is frequently associated with other metals and sulphur, in various mineral products. Many soils contain arsenic ; vegetables growing in such soils may contain traces of that body, which is more abundant in their seeds. The soil may also be rendered arsenical artificially, as when various chemical products are thrown upon the ground. Contamination of water may occur under those circumstances. Some valuable mineral waters, more especially the ferruginous waters, contain minute quantities of the poison, owing to their passage through arseniferous strata. It has also been found in sea water. It has even been lately asserted that arsenic is a normal constituent of the human body. Taking these things into consideration, I thought that the mere detection of traces of arsenic would not be sufficient to prove that any sample of beer was actually dangerous to health. No authoritative statement could be found showing that pure beer should be absolutely free from arsenic ; on the other hand, it was quite conceivable, on the ground of general knowledge, that minute traces of arsenic might occur in beer even prepared from the best material, and that this must have, in fact, occurred at all times since beer began to be brewed from malt and hops.
What is a Dangerous Amount of Arsenic in Beer? The first thing to do was therefore to determine what amount of arsenic might reasonably be considered dangerous for a man taking a considerable amount of beer, and, if that proportion was reached, it was obvious, first, that no argument based on the general prevalence of arsenic could shake the conviction that the presence of that body in the incriminated beer was harmful; and, secondly, that the presence of a harmful amount of arsenic in beer could not be accounted for by the use of natural products which had been employed in the manufacture of beer for generations without giving rise to any striking outbreak of disease, but that come substances capable of becoming loaded with arsenic had lately been used by a certain number of brewers, either in the treatment of ordinary brewing material or as substitutes for some important article. I have, therefore, attempted to fix a standard of the minimum dose that might reasonably be considered harmful, on the supposition that half a gallon of beer was consumed daily.

About $0.04 \mathrm{gr}$. of arsenious acid is about the minimum dose administered daily for medicinal purposes (this dose corresponds to about 2 minims of Fowler's solution twice a day). Such a dose may be taken for a considerable time by most people without any bad effects, but taking idiosyncrasy into account nobody should take such a quantity of arsenious acid daily and more or less indefinitely without knowing it or without medical advice. I therefore felt justified in conconsidering that any beer containing about $0.08 \mathrm{gr}$. of arsenious acid per gallon (that is, I part of $\mathrm{As}_{2} \mathrm{O}_{3}$ per 875,000 parts of beer) should be condemned. This could be done without, in the short time at my disposal, attempting to determine whether traces of arsenic were permissible in beer or not.

\section{Reinsch's Process.}

The selection of a proper method for the detection of arsenic in beer was not less important. The two most reliable qualitative methods are undoubtedly the Marsh's and the Reinsch's, but neither of these had been extensively used for the purpose of estimating the amount of arsenic in beer and some of the organic substances we had to deal with. At any rate, references to such examinations were not easy to find." Several comparative trials showed me that the Reinsch's test was eminently applicable to the detection of arsenic in beer, glucose, hops, and all the other organic and inorganic compounds I had to deal with. With regard to the Marsh's test, it was inapplicable to beer which had not been previously treated so as to break up the organic matter. I therefore thought that if the first method could be made to reveal sufficiently small quantities of arsenic in beer, and be used to estimate approximately the quantity of the poison in a moderate amount of fluid, it would be preferable

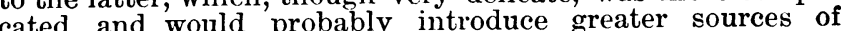
experimental error. By conducting the Reinsch's test with care, I found that it was easy to detect the presence of arsenic in 100 c.cm., and even as little as $20 \mathrm{cccm}$., of some of the suspected beers. On the other hand, no distinct trace of arsenic could be found in as much as $200 \mathrm{c.cm}$. of beer brewed in Bavaria, where the use of malt substitutes is prohibited by law.

The next thing to ascertain was the amount of arsenic that must be present in beer in order that the test should give unmistakable results. From a number of samples of beer, which had carefully been tested for the presence of arsenic, one was selected in which no trace of the poison could be found. With this beer standard solutions, containing respectively $0.002,0.001,0.0008$, $0.0006,0.0004,0.0002,0.0001,0.00005$ gram of arsenious acid per . Then $100 \mathrm{c.cm}$. of each one of $100 \mathrm{c.cm}$. of beer were made. Then solutions were tested by Reinsch's method, and it was found that a sublimate composed of small but clear crystals of arsenious acid could be obtained from the solution containing 0.0001 gram of arsenious acid per cent. (I part per million) without any previous concentration of the beer. The amount of sublimate obtained was just enough to obtain confirmation of its nature by the production of the yellow arsenite of silver. It will be noticed that the test demonstrates easily a quantity of arsenic smaller than that which I have given above as objectionable, and 
certainly not to be tolerated. Instead of adopting the standard of $I$ in 875,000 , based on the medicinal dose, I thought it best to adopt the more stringent standard of $\mathrm{I}$ in $\mathrm{I}, \infty 00, \infty 00$, which is elose enough to the former. This amount of arsenic is higher than that which we have so far found in beers brewed from ordinary malt and hops. On the other hand, this quantity is considerably below that found in beers brewed from arsenical glucose. It will be understood that the standard I have adopted for the present is purely artificial, and that by Reinsch's method it would be possible to fix a much more stringent standard if mecessary, for there would be no difficulty in discovering by this test I part in 5,000,000, in 10,050,000, or even le $>\mathrm{s}$, by increasing the quantity of, or concentrating the fuid under examination.

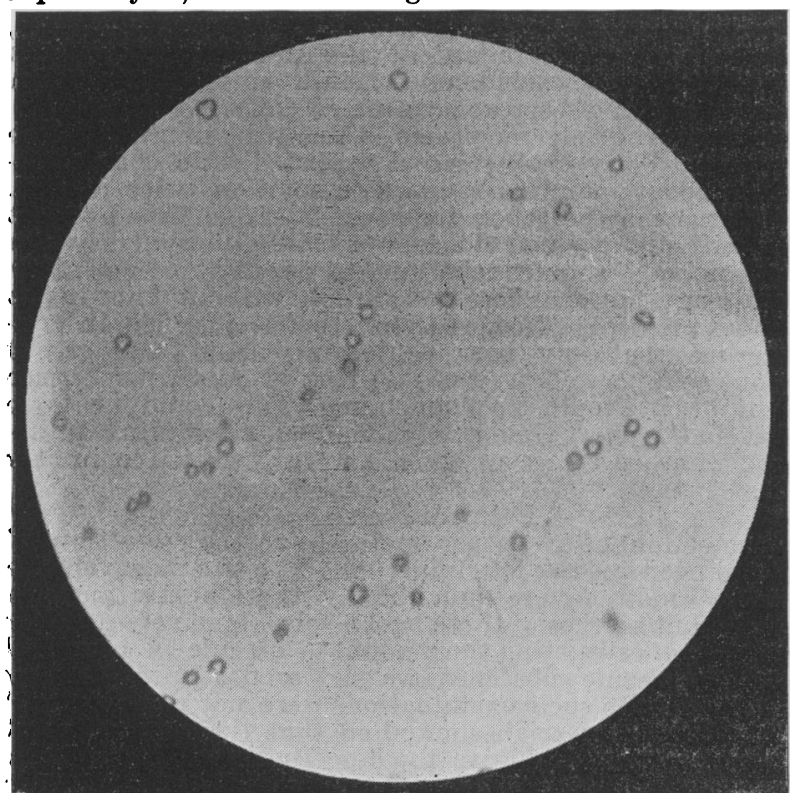

F $\Rightarrow$ I. Sublimate obtained from $\frac{1}{2}$ of the col per used in testing 50

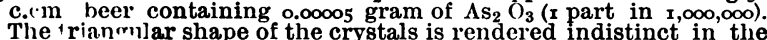
photograph but is very clear in the preparation. The crystals were of uniform size.

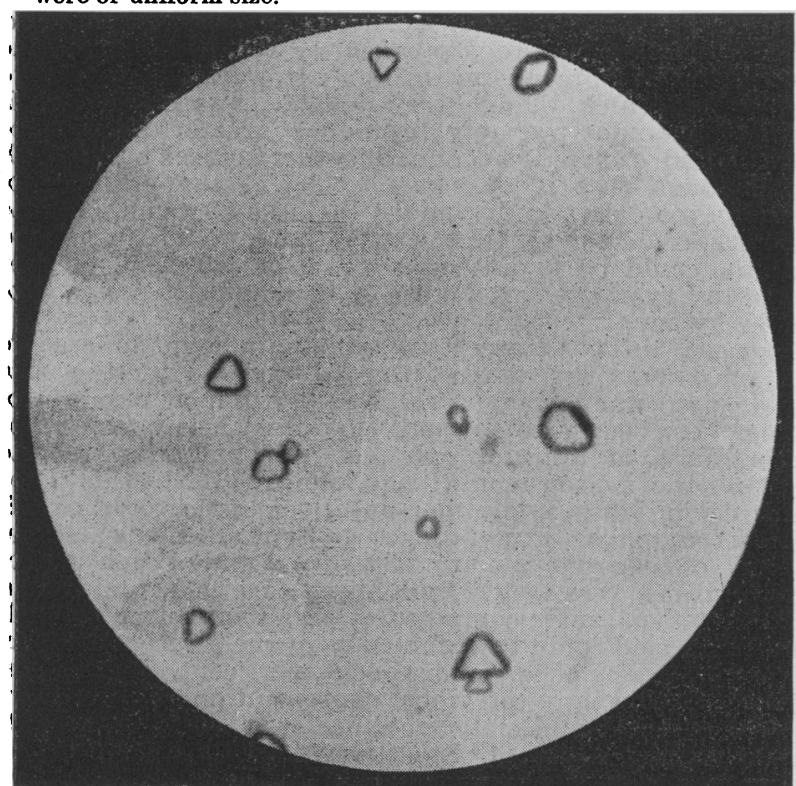

Wig. 2.-Sublimate obtained from $\frac{1}{5}$ of the copper-used in testing xoo c.cm. of beer containing 0.00 r gram of arsenious acid (r part

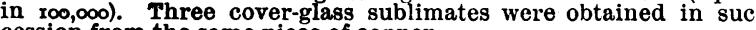
cession from the same piece of copper.
To estimate rapidly quantities of arsenious acid above $\mathbf{I}$ part in I million, it is only necessary to reduce the quantity of beer used until one finds the smallest amount that will give a deposit on copper, ${ }^{*}$ yielding a sublimate of arsenious acid corresponding to the standard deposit obtained from $100 \mathrm{c.cm}$. of the such a result, it may be assumed that there are at least 4 parts of arsenious acid in $1,000,000$ parts of beer. To test whether this inference was correct, I had a number of experiments made with various quantities of beer containing definite quantities of arsenious acid, and found that the amount of arsenic deposited corresponded to the amount of arsenic present in the quantity of beer used. It was suggested to me that, instead of using smaller and smaller quantities of beer, one should rather dilute the beer with water until the minimum limit was

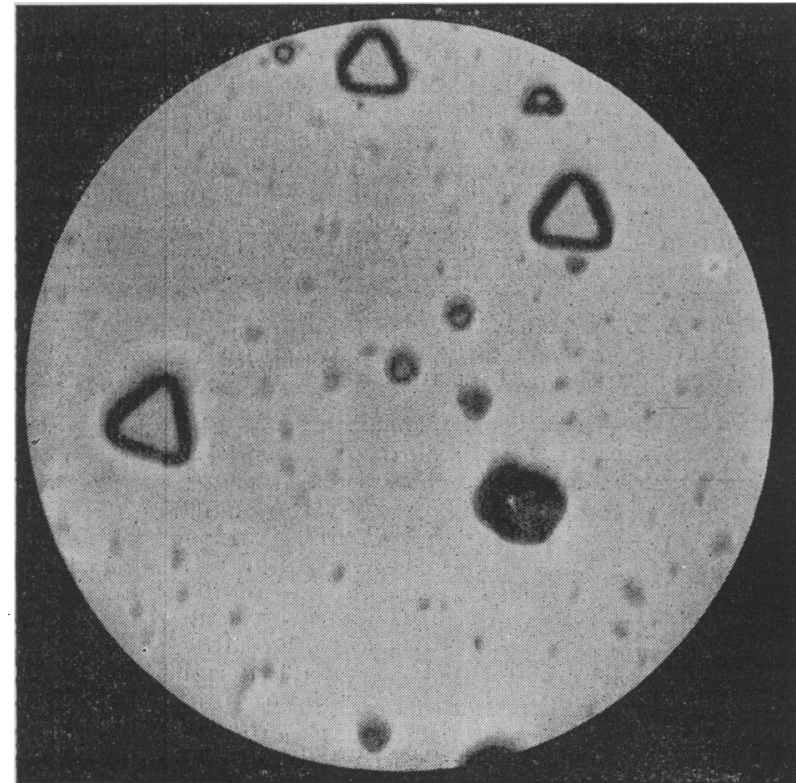

Fig. 3. - Sublimate obtained from $\frac{1}{6}$ of the copper used in testing $x_{5}$ grams of arsenical glucose dissolved in roo c.cm. of water.

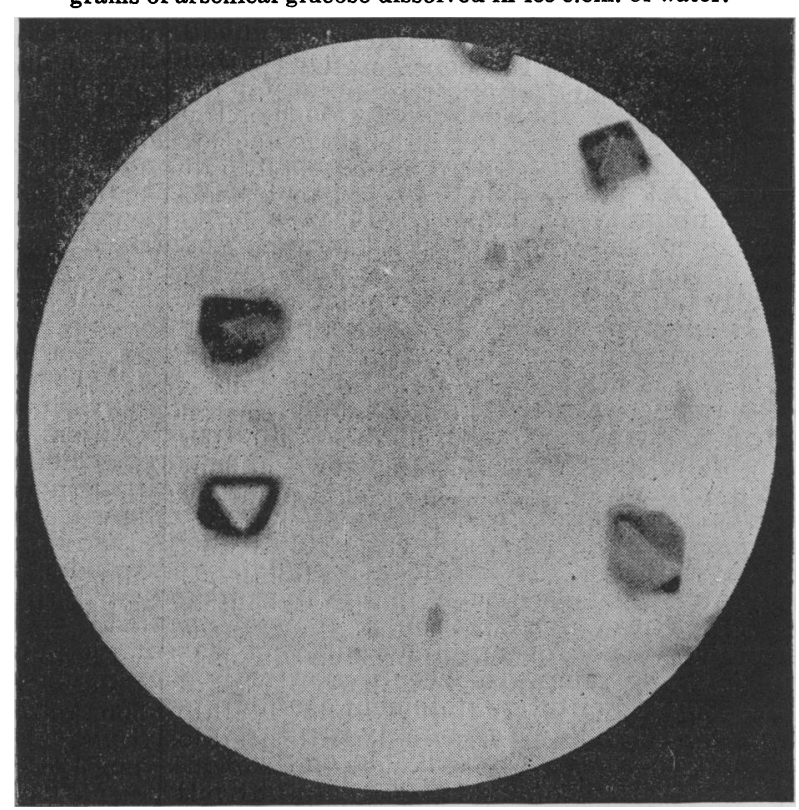

Fig. 4.-Margin of one of ; the 6 films obtained from the copper used in testing $2 \mathrm{c} . \mathrm{cm}$. of the sulphuric acid used in the preparaion of the above glucose. 
attained. I thought, however, that the conditions under which the precipitation of arsenic was taking place would be altered by dilution, and the results obtained less comparable; this was found to be actually the case.

To prevent any misconception I must point out that I have good reasons to believe that during the time ( $\frac{1}{2}$ to $\mathrm{I}$ hour) the copper is left in contact with the boiling acidified beer the arsenic present in that fluid is not entirely precipitated. But from experiments made with standard solutions it was clear that the deposit obtained bore a definite ratio to the amount of arsenic present in the fluid, so that it is possible to estimate that amount empirically. In estimating the amount of arsenic in glucose, invert sugar, sulphuric acid, malt, hops, and other products, the same method was adopted with slight modifications. To utilise the results of the analyses with the object of finding where the arsenic came from, it is evident that account must be taken of the proportion of the various ingredients used in brewing the quantity of beer under examination. Supposing that an arsenical beer was entirely brewed from malt, the malt should contain at least four times more arsenic than the beer prepared with it, since the proportion of malt used may be said roughly to be one-fourth of the total amount of beer produced from it. We have not, however, found yet any sample of malt (collected before the onset of the beer scare) containing more than a small fraction of the arsenic present in arsenical beer to which cases of poisoning had been traced.

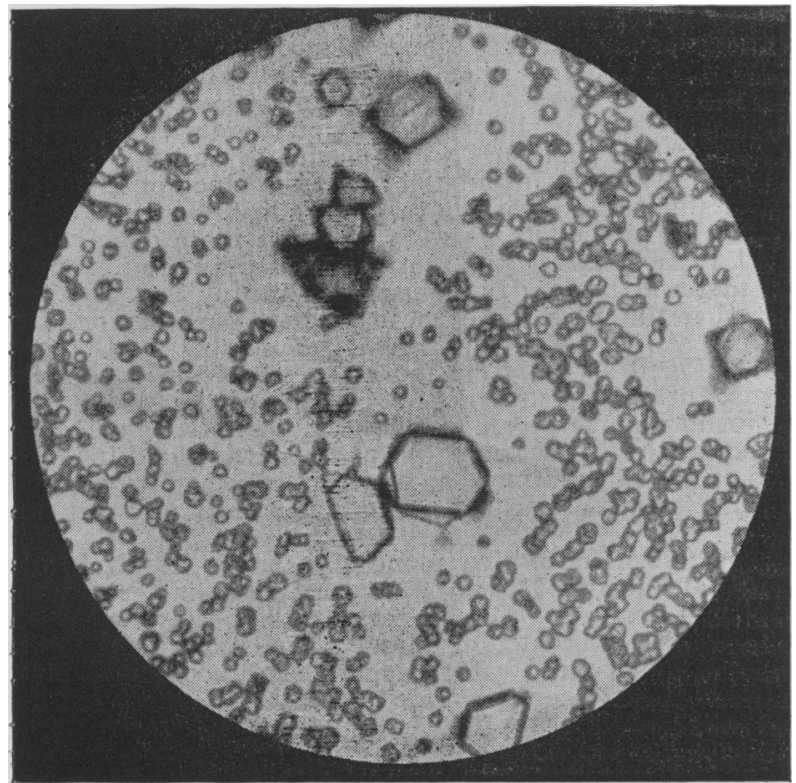

Fig. 5.-Intermediate rart of the same film as Fig. 4.

With regard to the glucose and invert sugar, the amount of arsenic found in some samples was more than sufficient to account for the dangerous contamination of the beer. The sulphuric acid used in the factory from which all the arsenical glucose we have examined came contained so much arsenic that it was actually saturated. A deposit of arsenious acid (or some arsenical compound formed in the presence of sulphuric acid) was actually found at the bottom of the bottle containing the acid sent to me for examination. By the modification of Reinsch's method just described we found that the amount of arsenious acid in one sample of $\mathrm{H}_{2} \mathrm{SO}_{4}$ was about 2 per cent.

It is not my intention to enter here into fuller details, which will be recorded in Dr. Tattersall's final report.

\section{Practical Notes}

With regard to the carrying out of the test a few particulars may not be out of place here. For detection of arsenic the following quantities were taken:

roo or 200 c.cm. of beer.

I 5 to 30 grams of malt.

ro to 5 grams of glucose.

ro to 15 grams of invert sugar.

$\frac{1}{2}$ to 2 c.cm. sulphuric acid. hydrochloric acid (x part of pure hydrochloric acid to 5 parts of water). The same quantity (or a little more when necessary) of the same strength of dilute hydrochloric acid was added to insoluble substances, such as hops and malts. The sulphuric acid was neutralised with pure ammonia before being tested. In the case of the beer, I part of hydrochloric acid was added to 5 parts of the fluid.

In nearly all the estimations the piece of copper used measured about three-quarters of an inch by three-eighths of an inch, and was divided into five strips. Both smaller and larger quantities were taken for special purposes. I have finally adopted two pieces of pure copper foil, measuring each exactly $\frac{1}{4}$ in. square. The fluid was kept gently boiling from three-quarters of an hour to one hour. The blackening of the copper is no sure index of the presend of an coperi rains af quarities of ar of arsenic may an quanties of arser employed were found to answer atory were tat tubes which had kindly been given by Dr. Dixon Mann; but, although the flat tubes are very convenient for microscopical examinations, much smaller cylindrical thin tubes, measuring under in. in diameter, were found to be better for the discovery of very in the application of heat

In order to obtain crystals easily examined under the highest powers of the microscope I have found it advantageous to cause the crystals to form copper foil. The cone measures in in diameter and $\frac{1}{2}$ in. in height An copper foil. The cone measures in. in diameter and $\frac{1}{2}$ in. in height. An iron plate $\frac{1}{8}$ in. thick and 4 in. In diameter, with a central perioration 8 in. in diameter, is used to support the cone, the apex of which projects rests on the upper surface of the iron plate. The open basis of the cone is closed with a cover glass 7 in. in diameter.

To test the nature of the deposit on a piece of copper the cone is first heated to redness. When it is cold the piece of copper to be tested is placed quite at the apex of the cone-if the piece of copper is large, it should be divided into small square or triangular pieces not more than $\frac{1}{8}$ in. in diameter; the opening of the cone is then closed with the perfectly clean cover glass.

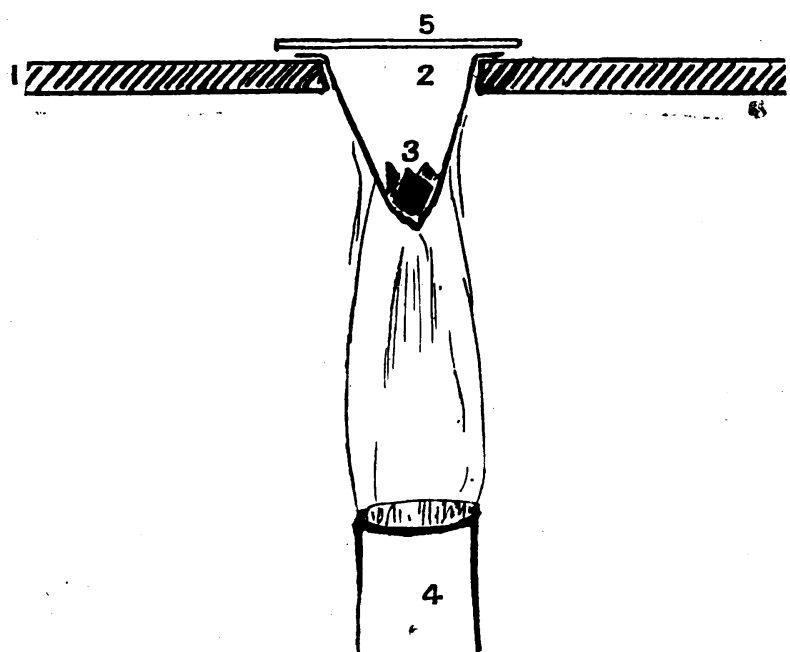

Fig. 6.-Dr. S. Delépine's apparatus for the oxidation of arsenic and . Thick iron plate, which must not be allowed to get very hot during the heating of

Cone made of " electric" copper foil absolutely free from arsenic.

3. Small pieces of copper covered with deposit of arsenic and placed in the portion of the cone which must be brought to a dull-red heat by means of the flame from a Bunsen burner (4). A smaller flame may be used with advantage until sufficient practice has been acquired. 5. Cover glass.
The apex of the cone is then heated to dull redness by means of a small Bunsen flame, the cover glass is watched. and as soon as a distinct sublimate
has formed the flame is removed, and the cover glass is taken with a pair has formed the flame is removed, and the cover glass is taken with a pair to obtain a large number of small but very clear crystals of arsenious acid

t This method resembles closely one recommended by a Committee of the National Health Society. (BRITISH MEDICAL JoURNAL, r883, i, p. 1220 ; and Luff, Textbook of Forensic M the form I have adopted having for its object to allow the complete volatilisation and oxidation of the arsenic deposited on the copper without any overheating of the cover glass. I am not aware that this method has been used for quantitative purposes previously. I propose it as one which may be used to determine rapidly and approximately the amount of arsenious acid, free or in combination, when Reinsch's process is applicable. The narrow sublimation tube is more specially suitable for the detection of minute traces, and should always be used in a preliminary test, one of the two pieces of copper being employed for the purpose. The cover-glass method is somewhat less delicate, but is more convenient for estimating quantities exceeding one part in one million. 

from 5o c.cm. of beer to which 0.00005 gram ( $=0.007$ grain) of the cone when the quantity of arsenic is less than so milligram, but examination under a higher power of the microscope is more difficult. The cover-glass method allows of the use of an oil immersion lens for the detection of quantities under results which are specially useful for quantitative estimations.

I have not entered into all the precautions to be observed in carrying out the test, because complete accounts will be found in Dr. Taylor's, Dr. Dixon Mann's, and Dr. Luff's excellent treatises. With regard to the carrying out of many of the experiments, I have to thank my friend Dr. Coutts, whose skill and enthusiasm have been of the greatest use to me.

Photographs of arsenious acid sublimates $\times 75^{\circ}$

$$
\text { REFERENCES. }
$$

1 BRITISH MEDICAL JOURNAL, December Ist, 1900. 2 Watts's Dictionary of Chemistry, r888, i, p. 302.
Physiologie, 1895, i, p. 674. 4 Clouet, Ann. d'Hygiene Publique, xlix, 1878; Wynter Blyth, Foods, etc., 1896, p. 542.

\section{A NOTE ON THE VALUE OF INOCULATION AGAINST ENTERIC FEVER.}

BY Colonel HENRY CAYLEY, F.R.C.S.,

Late Officer in Charge of the Scottish National Red Cross Hospital, South Africa.

THe question of the value of antityphoid inoculations in producing immunity against enteric fever has attracted much attention, and its importance, especially in the case of soldiers, can hardly be overestimated. The statistics of the army in South Africa will no doubt in due course be published, and must have great value, but, as everyone serving out there knows, the returns of patients treated in the hospital there are open to so many sources of error that any conlusions drawn from them will be very unreliable. On thesef cpoints I will not now enter, but $I$ think that the results o the inoculations of members of the staff and establishment of the Scottish National Red Cross Hospital serving in South Africa are of interest sufficient to warrant their publication.

The first section of the hospital, consisting of 61 personsofficers, nursing sisters, and establishment-left Southampton on April 21st, 1900, by steamer Tanjore for South Africa. During the voyage out all except 4 of the 6r personnel of the hospital were inoculated twice at an interval of about ten days. The inoculations were performed by the ship's surgeon, Dr. Wortabet, who performed the operation with the greatest care, and measured the dose of the virus for each case with exactness. The injections were all made in the flank, and were all followed in from two to eight or ten hours by marked symptoms, both local and constitutional, lasting from two to four or five days.

In many cases the symptoms were just as severe after the second as after the first inoculation. This would seem to show that it takes more than ten days before any immunity is established. Two of the 7 nurses were not inoculated because they had both suffered from enteric fever, and 2 of the orderlies were only inoculated once.

The material for inoculation I had received from Professor Wright at Netley a few days before we embarked, and it was quite fresh. I may add that, out of about 300 troops on board, chiefly volunteers and militia, nearly 100 were persuaded to be inoculated.

Immediately we reached the Cape the hospital was sent up to Kroonstadt, in the Orange River Colony, and remained then as a stationary hospital till the middle of 'October. During this period there were always many cases of enteric under treatment in the hospital. Further, some of the medical officers and student orderlies had charge of the Kroonstadt Hotel temporary hospital, which was crowded up with enteric cases, and the nursing sisters for three weeks did duty in the military hospitals at Bloemfontein in May and June, when enteric fever was at its worst. There was not a single case of enteric amongst the personnel of this first section of the hospital.

The second section of the hospital, medical officers, nurses, and establishment-82 in all-left Southamption in May, 1900 . On board ship nearly all were inoculated, but many of them only once. The material for inoculation had been on board for some time, and was not so fresh as in the first instance. Of this second section, one nurse had enteric at Kroonstadt. She was the only one, out of a total of 36 nurses, who suffered from enteric, and she was the only nurse who was not inoculated, excepting the 2 who were protected by a previous attack of enteric. A third section of the hospital, consisting of 4 medical officers and 16 nurses, went out in July; they were all inoculated, and none of them had enteric.

Of the second section 5 orderlies had enteric fever at Kroonstadt, of whom 2 died. Of these 5 there were 2 inoculated (once) and 3 non-inoculated. Of the 2 who died $\mathrm{I}$ had been once inoculated, the other had not been inoculated.

At the end of August Dr. Dodgson, who was deputed by the Director-General of the Army Medical Department to examine the results of the antityphoid inoculations, came to Kroonstadt, and was good enough to examine the blood of a number of the staff and establishment by Widal's test.

Of the first section who had been inoculated four months previously, he tested the blood of 23 . Of these, 21 gave good reaction with dilutions ranging from $I$ in 40 to $I$ in 500: 2 gave only slight reaction-these were the two orderlies who had only been once inoculated.

Of the second section of the hospital who had been inoculated only three months previously, the blood of 22 was tested. Of these i I gave no reaction, 9 gave very slight reaction, and only 2 gave good reaction.

It would appear from the above that the members of the first section were much more fully protected against enteric than those of the second section. None of the first section got enteric, though very much exposed to its influences, also the blood of all those examined showed good reaction to Widal's test. Among the second section there were 5 cases' of enteric, of whom 3 had not been inoculated, and the other 2 had been only once inoculated; also the blood of 22 tested gave very slight or no reaction to Widal's test. It is not quite clear why the inoculations were so much more effective in the first than in the second section. Probably the freshness of the material had an influence; also I think that with the first section the dose for each individual was more exactly measured. As far as I could learn, the members of the first section suffered at the time more severely from both local and constitutional disturbances than those of the second.

The results above given are, I think, very strong evidence in favour of the protective power of the antityphoid inoculation, when the inoculations are very carefully performed, and they point to the necessity for two inoculations at a suitable interval.

During the period of five months that the hospital was stationed at Kroonstadt there were 92 admissions for enteric fever, and II deaths. Of the 92 cases, 15 said they had been inoculated, a few were doubtful, and about 70 had not been inoculated. Of the II deaths, I had been inoculated once; the others had not been inoculated.

From these figures I do not think that any conclusions or even reliable inferences can be drawn. One knows nothing as to the relative number of men inoculated or not in the different corps from which the patients came. Some of the men on admission were not in a condition to say whether they had been inoculated or otherwise. In not a few cases the patients did not know whether they had been vaccinated or inoculated against enteric, as on some of the ships the inoculations against enteric were performed on the arm. There are many other sources of error which I need not go into, but I fear that any statistics bearing on this question, derived from the hospitals in South Africa, will not be of any great value. At the same time, I feel convinced from the cases I saw in the hospital that the attacks of enteric were as a rule mucy milder in the inoculated than in the non-inoculated, and the duration of the disease was much less.

Children's Hospital, Notringham.-On December 17 th, a new building was opened at Nottingham for the Children's Hospital. The building is an adaptation of a large country house, situated in $3 \frac{1}{2}$ acres of ground near the centre of the town, both being the gift of Mr. T. J. Birkin, J.P., who also contributed $£ 3,000$ towards the necessary alterations and additions. A new pavilion ward, with smaller wards and an operating theatre have been added with a detached outpatient department. 